\title{
27 Der Krimkrieg: Die Ereignisse auf der Halbinsel
}

\begin{abstract}
Ruined Pompeii is in good condition compared to Sebastopol. Here, you may look in whatsoever direction you please, and your eye encounters scarcely anything but ruin, ruin, ruin! - fragments of houses, crumbled walls, torn and ragged hills, devastation everywhere! It is as if a mighty earthquake had spent all its terrible forces upon this one little spot. For eighteen long months the storms of war beat upon the helpless town, and left it at last the saddest wreck that ever the sun has looked upon. ${ }^{1}$
\end{abstract}

Der später als Schöpfer der literarischen Figuren Tom Sawyer und Huckleberry Finn weltberühmt gewordene US-amerikanische Schriftsteller Mark Twain (18351910; bürgerlicher Name Samuel Langhorne Clemens) schilderte seinen Eindruck von Sevastopol', das er mehr als ein Jahrzehnt nach Ende der Belagerung im August 1867 in Augenschein nahm. Als Reporter im Auftrag der Zeitung „San Francisco California Alta“ nahm er an einer Vergnügungsreise mit dem Schiff „Quaker City“ teil und veröffentlichte seine Eindrücke 1869 unter dem Titel „The Innocents Abroad - Or the New Pilgrims' Progress“ in Buchform. ${ }^{2}$

Die Spuren der „Stürme des Krieges“, so entnimmt man Twains Zeilen, waren auch mehr als eine Dekade nach Kriegsende noch nicht beseitigt worden. Die „hilflose Stadt“ hatte sich im Krimkrieg zwar nicht achtzehn Monate, wie der Amerikaner schrieb, aber immerhin 349 Tage - vom 13. September (26. September) 1854 bis zum 27. August (9. September) 1855 - im Belagerungszustand durch alliierte Truppen befunden. Der, beginnend mit Tolstoj (Kapitel 26), als heldenhaft bezeichnete Widerstand der Frauen, Männer und Kinder der Stadt stellt in der Geschichte der Krim seit 1783 wohl den Moment dar, welcher im Bewusstsein vieler Russinnen und Russen am nachhaltigsten die emotionale Aneignung nicht nur Sevastopol's, sondern der ganzen Krim befördert hat. Durch eine zielgerichtete geschichtspolitische Lancierung und komplexe erinnerungskollektive Prozesse wurde die Halbinsel mit Rekurs auf die ,349 Tage' zur unveräußerlichen Heimat nicht nur für die auf der Krim lebende russische Bevölkerung, sondern in einem übergeordneten Sinne auch für ,das' russische Volk in anderen Landesteilen. Das belagerte Sevastopol' wurde zur Chiffre des Ruhmes und des Leidens einer ganzen Nation - und zwar sowohl in zarischer als auch in sowjetischer Zeit.

1 Twain (1966), 284.

2 Zu Twains Russlandreise und seiner Haltung zum Zarenreich vgl. Fuchs (2011).

๑ OpenAccess. ( 2020 Kerstin S. Jobst, publiziert von De Gruyter. (cc))BY Dieses Werk ist lizenziert unter der Creative Commons Attribution 4.0 International. https://doi.org/10.1515/9783110520620-029 
Und das gilt noch heute, wie die in der Russländischen Föderation verbreitete Begeisterung für die Annexion der Krim 2014 belegt. $^{3}$

Wenn auch der Wiederaufbau der zerstörten Gebäude Twain (und anderen, hier nicht genannten Autoren) zufolge auf sich warten ließ, so war bereits unmittelbar nach Kriegsende die Schaffung einer materiell greifbaren heroisch-militärischen Infrastruktur mit Denkmälern und Erinnerungstafeln begonnen worden, anfänglich übrigens in privater - nicht staatlicher - Initiative. ${ }^{4}$ Später erhielt Sevastopol' zahlreiche Ehrenzeichen: 1954 wurde die Stadt mit dem „Orden des Roten Banners“ ausgezeichnet, 1965 erhielt sie den Titel gorod-geroj (Heldenstadt), womit sowohl der Ereignisse im sog. Großen Vaterländischen Krieg (vgl. Kapitel 33) als auch im Krimkrieg gedacht wurde. Der sowjetische Militärhistoriker E.V. Tarle (1874-1955) benannte folgerichtig sein 1954 erschienenes Werk „Stadt des russischen Ruhms. “5 Nach dem Zweiten Weltkrieg nahm die Stadt Sevastopol' auch administrativ eine Sonderrolle ein: Wegen ihrer militärischen Bedeutung durfte sie selbst von Sowjet-BürgerInnen von 1948 bis in die 1990er Jahre hinein nur mit Sondergenehmigung besucht werden. Sie verfügte über ein selbst zu verantwortendes Budget, was innerhalb der stark zentralistischen UdSSR besonders war. ${ }^{6}$ Nur mit Moskau und Leningrad zu vergleichen war auch ihr Status als „Stadt föderalen Ranges. “7 Seit der russischen Annexion 2014 genießt sie unter den insgesamt 85 Unionssubjekten der Russländischen Föderation übrigens erneut eine bevorzugte Position.

Der Nimbus des unter Potemkin neugegründeten Sevastopol's entstand nachhaltig erst durch die ,heldenhafte Verteidigung، im Krimkrieg. Ursprünglich war sie eine „militärische Zweckgründung“" „Erhabene Stadt“ (so die Übersetzung ihres Namens) doch ganz auf militärische Erfordernisse zugeschnitten: Zahlreiche Kasernen, Magazine, Arsenale, Werften und breite, gerade angelegte Straßen entstanden. Entgegen der Bevölkerungsstruktur in den meisten anderen Teilen der Halbinsel war Sevastopol' am stärksten slavisch (d.h. russisch-ukrainisch) geprägt, zum einen weil es als Gar-

3 Zur Genese des Sevastopol'-Mythos in der russischen und sowjetischen Geschichtspolitik vgl. Plokhy (2000).

4 Vgl. hierzu Semin (1955), 152-169, der einen Überblick über die unmittelbar nach dem Ende der sog. oborona (Verteidigung) begonnenen erinnerungspolitischen Maßnahmen aus den Reihen „des Volkes“ gibt. Die von der Administration und der Zarenfamilie initiierten Aktionen zur Ehrung der Gefallenen bezeichnete er zeittypisch als „heuchlerisch“, $154 \mathrm{f}$.

5 Tarle (1954).

6 Vaneev (1983), 22.

7 Sasse (2007), 229.

8 Jena (2001), 188, zum Wirken Potemkins bei der Stadtgründung besonders 188-190. 
nisonsstadt primär von Soldaten bewohnt war und viele sog. fremdstämmige männliche Untertanen (einschließlich der Krimtataren) vor der Einführung der allgemeinen Wehrpflicht von 1874 nur auf freiwilliger Basis in der Armee dienten, zum anderen weil der Anteil von Nichtslaven aus Sicherheitserwägungen gering gehalten wurde. Vor 1853 zählte es knapp 40.000 Einwohner, wobei der Anteil der weiblichen Bevölkerung überproportional niedrig war, was in Militärstädten, wo sich zumeist nur die Familienangehörigen der Soldaten ansiedelten, die Regel war. ${ }^{9}$ In- und ausländische Besucher zeigten sich bereits vor dem Krimkrieg von der Lage und der Architektur Sevastopol's beeindruckt, nach 1855/56 priesen nicht wenige auch das ,russische Heldentum', also die Standhaftigkeit während der Belagerung. ${ }^{10}$ Russischsprachige Autorinnen und Autoren maßen die ,349 Tage‘ häufig an biblischen oder antiken Ereignissen. Nicht nur bei Twain war Pompeji, welches bekanntlich durch den Ausbruch des Vesuvs 79 n. Chr. zerstört worden war und das zu einem kulturübergreifenden Synonym für Katastrophen wurde, eine Vergleichsgröße: In einer in den 1880er Jahren veröffentlichten Befassung verglich ein russischer Autor die Reaktionen der BewohnerInnen beider Städte im Angesicht des Schreckens. Während die Bevölkerung der römischen Stadt in panischer Angst geflohen sei und sich passiv der unumstößlichen Kraft der Naturgewalten ergeben hätte, wehrten sich im belagerten Sevastopol' „die Menschen gegen die feindliche Macht und entfesselten selbst Naturgewalten, in dem stolzen Wunsch am Ende alles zu vernichten, was zu verteidigen unmöglich war."11 Auch Ausländer, die nach 1855 in großer Zahl die Stadt als sog. BattleField-Reisende besuchten, zollten dem Beharrungswillen ihrer BewohnerInnen häufig mit transzendenten Bezügen Anerkennung: Sevastopol' sei zu Recht, so ein deutschsprachiger Autor, „zum russischen Sanctuarium der russischen Armee, der russischen Nation“ geworden. ${ }^{12}$

Während also die Belagerung Sevastopol's im Krimkrieg ZeitgenossInnen und in der Forschung präsent ist, trifft dies auf die Ereignisse auf der übrigen Halbinsel weitaus weniger zu. Kaum einmal wurden das Leben und die Lage abseits der Kampfgeschehnisse konkret untersucht. Abgesehen von Arsenij Markevičs (1855-1942) Beschreibung des Taurischen Gouvernements, die aus dem Jahr 1905 stammt, lagen bis vor kurzem keine seriösen Untersuchungen über die Geschichte

9 Nach Seaton (1977), 106, hatte sich die Bevölkerungszahl bis 1854 sogar auf 45.000 erhöht. Dies ist vermutlich mit dem in Kriegszeiten höheren Anteil an Militärs zu erklären.

10 Vgl. dazu Jobst (2007b), 354-367 und 380-406.

11 Vjazmitinov (1882), 62.

12 Schweiger-Lerchenfeld (1887), 204. 
der Halbinsel im Krimkrieg vor. ${ }^{13}$ Sie sind wichtig, um die schon angesprochenen krimtatarischen Migrationswellen in den folgenden Jahren zu verstehen: Die Halbinsel war 1854 im Verlauf des Septembers zum Kriegsschauplatz geworden. Es begann die von Zeitgenossen „das Chaos“ genannte Phase, die nicht zuletzt eine Folge unglücklicher militärischer Entscheidungen des damaligen Oberbefehlshabers über die Krim-Truppen, Fürst Alexander S. Menšikov (1787-1869), gewesen war. Der altgediente, schon während der Napoleonischen Kriege aktive Militär hatte nämlich die in der Rückschau fatale Entscheidung getroffen, die russischen Truppen in Sevastopol' zu massieren und damit die anderen weitläufigen Küstenabschnitte der Halbinsel möglichen - und dann auch eingetretenen - gegnerischen Angriffen schutzlos auszuliefern. ${ }^{14}$ Damit sind mit seinem Namen gleich zwei schlechte Entscheidungen im Zusammenhang mit dem Krimkrieg verbunden: Anfang 1853 war er bekanntlich von Zar Nikolaus I. (17961855) nach Istanbul entsandt worden, um dem Sultan die russischen Forderungen zur Lösung des sog. Mönchsgezänks zu überreichen. Die von Menšikov einkalkulierte Ablehnung Istanbuls hatte St. Petersburg den Vorwand zur Auslösung des Konflikts gegeben.

In jedem Fall hat sowohl seine Strategie des alleinigen Schutzes der Hafenstadt als auch die Entscheidung, zivile Behörden und alle staatlichen Stellen weitestgehend nach Norden auf das Festland zu evakuieren, Angst bei der Bevölkerung ausgelöst. Die schutzlose Bevölkerung, gleich welcher Nationalität, flüchtete panisch. Verschlimmert wurde die Situation noch durch Anweisungen, Lebensmitteldepots $\mathrm{zu}$ zerstören, um sie nicht in gegnerische Hände fallen $\mathrm{zu}$ lassen, was zu Versorgungsengpässen führte. Wer nicht (was häufig tatarische BewohnerInnen betraf) über genügend Eigenmittel verfügte, um auf eigene Faust nach Norden zu gelangen, geriet in bedrohliche Lagen. Plünderungen und Gewalt waren die Folge. ${ }^{15}$ Der Versuch der Behörden, die Situation mittels des Einsatzes von Don-Kosaken zu kalmieren, bewirkte das Gegenteil, weil sich diese an Räubereien beteiligten und nicht selten mit unangemessener Gewalt gegen vermeintliche und tatsächliche Plünderer vorgingen. Es kam zu zahlreichen Verhaftungen auch Unschuldiger sowie $\mathrm{zu}$ Vergewaltigungen einheimischer Frauen. ${ }^{16}$

Die Alliierten wiederum hatten sich - auch dies mutet ,modern' an - Gedanken darüber gemacht, ob man nicht den muslimischen Untertanen des Zaren

13 Markevič (1994). Erst durch die Beiträge Mara Kozelskys, auf die ich mich im Folgenden stütze, hat sich dies geändert. Vgl. besonders: Kozelsky (2018).

14 Kozelsky (2014), 168f.

15 Kozelsky (2014), 168f.

16 Gercen (1861), 973. 
konkrete Angebote zur Kollaboration machen sollte, um die zarische Kampfkraft ,von innen‘ zu schwächen. So veröffentlichte der französische Oberbefehlshaber Armand Jacques Leroy de Saint-Arnaud (1798-1854) eine Proklamation, die den von der russischen Obrigkeit verlassenen Krim-MuslimInnen Schutz und Nahrung versprach. Es nimmt kein Wunder, dass einige KrimtatarInnen dieses Angebot annahmen. Dies zumal in Evpatorija, wo die Alliierten bei ihrer Anlandung im März 1854 außer einigen verletzten russischen Soldaten keine Mitglieder der zarischen Armee vorfanden. Stattdessen vergegenwärtigten sie „a real humanitarian crisis“, da an die 40.000 geflohene tatarische Bauern unversorgt in der Stadt vegetierten. ${ }^{17}$ Dass es vor diesem Hintergrund zur partiellen, aber keineswegs massenhaften Zusammenarbeit muslimischer BewohnerInnen mit den Besatzungstruppen kam, darüber wunderten sich auch ZeitgenossInnen nicht. Einer von diesen war der im Exil lebende zarenkritische Schriftsteller Alexander Herzen (= Gercen; 1812-1870), der nur einer der ,Verteidiger‘ der krimtatarischen Bevölkerung vor dem Vorwurf der Kollaboration mit dem Gegner war. ${ }^{18}$ Mittelfristig fatal war, dass der ohnehin schon seit der Annexion von 1783 erhobene Vorwurf tatarischer Illoyalität neue Nahrung bekam. Dass tatarische Vertreter zu Beginn des Waffenganges wiederholt Adressen ihrer engen Verbundenheit mit dem Herrscherhaus veröffentlicht hatten und ihre Glaubensbrüder - und -schwestern - an ihren Treueeid gegenüber den Romanovs erinnerten, überzeugte einflussreiche russische Kreise immer weniger. ${ }^{19}$ Selbst der nach dem Tod seines Vaters den Thron besteigende sog. Befreier-Zar Alexander II. soll der krimtatarischen Bevölkerung gegenüber ablehnend gewesen sein und später dann ihre Auswanderung ausdrücklich gutgeheißen haben. Nicht zuletzt das Misstrauen des Zaren und Gerüchte über geplante Umsiedlungen (die es zum Teil gab) und Zwangstaufen - zu denen es nicht kam - sollen nicht wenige TatarInnen zur Ausreise ins Osmanische Reich veranlasst haben. ${ }^{20}$ Den Deportationen der krimtatarischen Bevölkerung im Mai 1944 ging eine lange Vorgeschichte des Misstrauens voraus (vgl. Kapitel 34).

In Kriegen kommt es regelhaft nicht nur zu Kampfhandlungen und damit zu Gewalt zwischen Kombattanten, sondern auch zwischen diesen und der einheimischen Zivilbevölkerung und sogar zwischen verschiedenen Gruppen innerhalb dieser. Auch geht die zivile oder militärische Verwaltung nicht selten gegen ,eigene Leute، vor, aus Angst vor Verrat und Kollaboration. Der Krimkrieg war hier

17 Figes (2010), 339.

18 Gercen (1861), 973f. Vgl. dazu auch Jobst (2007b), 239-243.

19 Kozelsky (2010), $168 \mathrm{f}$.

20 Jobst (2007b), 236. 
keine Ausnahme. In zeitgenössischen Debatten wurde u.a. darauf hingewiesen, dass sich tatarische Bauern in der Region Evpatorija gegen Exponenten der zarischen Macht aufgelehnt hätten, sobald sie der sich nähernden alliierten Schiffe gewahr geworden seien, und so die fremde Invasion begünstigt hätten. Zudem sollen sie flüchtende russische Menschen überfallen oder sogar ermordet haben. ${ }^{21}$ Nach der russischen Wiedereinnahme Evpatorijas unter General Korf kam es zu drastischen Vergeltungsmaßnahmen und Zwangsumsiedlungen nicht nur kollaborierender TatarInnen - die Rede ist von bis zu 20.000 -, ohne dass die Schuldfrage im Einzelnen geprüft wurde. ${ }^{22}$ Die Alliierten ihrerseits übten ebenfalls abseits des Schlachtfelds Gewalt aus; so etwa nach der Aufgabe Sevastopol's durch die russische Armee, als die französische Führung ihren Soldaten die Erlaubnis zur Plünderung gab bzw. erst verspätet dagegen einschritt. Auch im Osten der Halbinsel kam es zu Ausschreitungen: Das Vordringen in das Azovsche Meer und die Einnahme von Kerč' hatte für die alliierte Führung ursprünglich den Versuch dargestellt, Sevastopol' vom Nachschub abzuschneiden, was allerdings nicht gelingen sollte. In jedem Fall wüteten ihre Truppen dort in „betrunkener Raserei“ („drunken rampage“), verwüsteten die Stadt und drangsalierten ihre Einwohner, so Orlando Figes. Osmanische Soldaten sollen gemeinsam mit Tataren russische Läden ausgeraubt und zerstört sowie russische Frauen vergewaltigt haben. ${ }^{23}$ Englische und französische Soldaten wurden ebenfalls der Vergewaltigung bezichtigt, allerdings sollen diese unterschiedslos russische und tatarische Frauen geschändet haben. ${ }^{24}$ Im Einzelnen können die Ereignisse nicht verifiziert werden, festzuhalten ist, dass erstens militärische/ideologische Gegner häufig der Vergewaltigung bezichtigt werden, und dass zweitens sexuelle Gewalt gegen Frauen und Männer (obgleich in niedrigerer Zahl) bei militärischen Konflikten ein gängiges Kriegsmittel ist. Dabei geht es keinesfalls allein um sexuelle Befriedigung, sondern ebenso um die Demonstration von Macht. ${ }^{25}$ Weitere symbolisch wichtige ,Kapitalien` des Kriegsgegners werden verwüstet oder gestohlen, denn nicht erst im Zweiten Weltkrieg wurde Beutekunst - auch hier war der Krimkrieg durchaus ,modern' - zu einem Thema: Das französische Militär beispielsweise bemächtigte sich diverser auf der Halbinsel vorgefundener Kulturdenkmäler, darunter zwei Sphinxen und mehrere Basisreliefs. Es verschiffte und stellte sie im

21 Markevič (1994), 18-23

22 Gercen (1861), 974.

23 Figes (2010), 344.

24 Vgl. hierzu Trustam (1984), 175, sowie Edgerton (1999), 142.

25 Vgl. zur Diskussion des Topos Vergewaltigung Burgess-Jackson (1996), besonders 43-64. Eine Systematisierung der Kategorien Nationalismus, Sexualität und Vergewaltigung im Kriegsfall vgl. bei Pryke (1998). 
Sommer 1856 vor der Orangerie beim Palais des Tuileries aus. Pariser und Pariserinnen konnten sich so an der Beute und dem auch materiell greifbaren Sieg ihrer Truppen erfreuen. ${ }^{26}$

Allerdings musste zunächst der Krieg beendet werden. Auf der Krim ging er im Herbst 1855 allmählich zu Ende, wurde Sevastopol' doch im September 1855 nach massivem dreitägigem Beschuss geräumt. Vorher waren aber noch die Befestigungsanlagen von den russischen Verteidigern gesprengt worden, erst dann konnte die weitläufig zerstörte Stadt von französischen Truppen eingenommen werden. Trotz der Aufgabe Sevastopol's galt das Zarenreich in strategischer Hinsicht allerdings noch nicht als besiegt ${ }^{27}$, stand es doch etwa im Kaukasus noch auf osmanischem Gebiet. Bedeutend, auch im Hinblick auf die dann einige Monate später in Paris beginnenden Friedensverhandlungen, war die von General Nikolaj N. Murav’ëv-Karskij (1794-1866) geführte Belagerung der Festung Kars, die nach der Räumung Sevastopol's andauerte: Im November 1855 kapitulierten die osmanischen und englischen Verteidiger wegen einer Cholera-Epidemie und Lebensmittelknappheit. Trotzdem entschloss sich der erst seit kurzem regierende Zar Alexander II., auf das alliierte Friedensangebot einzugehen. Das Vordringen des ,neutralen', aber Russland offen mit Krieg drohenden Österreichs in die Donaufürstentümer war ein Grund dafür; ein anderer (vielleicht sogar wichtigerer) die Angst vor dem „unweigerlichen Bankrott“ bei Fortführung der Kampfhandlungen, wie Winfried Baumgart meint. ${ }^{28}$ Wie so häufig führten also pragmatische Überlegungen dazu, den Krieg zu beenden, da man sich diesen schlechterdings nicht länger leisten konnte. ${ }^{29}$ Hinzu kam die Angst vor bäuerlichen Unruhen, wovon erste Ausschreitungen nach der Einberufung der Landstände in frontfernen Reichsteilen kündeten. ${ }^{30}$ Groß war zudem das Misstrauen gegenüber den nichtrussischen Nationalitäten - nicht nur gegenüber der krimtatarischen, galten doch z. B. die Polen genauso als Unruhekandidaten wie die Völkerschaften im nördlichen Kaukasus. Dort führte das Zarenreich bekanntlich seit den 1820er Jahren einen ,kleinen', hybriden Krieg gegen die sog. Gorcy (Bergbewohner) unter der Führung Imam Šamils (1797-1871), der bis in die 1860er Jahre dauern sollte. Trotz des starken Misstrauens gegenüber den nicht-slavischen, religiös oder konfessionell divergenten Gruppen im Russländischen Reich: Der Krimkrieg war eigentlich ein Beleg für die Stabilität des Vielvölkerreiches, da es nicht zu um-

26 Senner (1999), hier 137 und 145.

27 Baumgart (1972), 11.

28 Baumgart (1972), 116

29 Nach Wegner (2002), XXIV, ist dies bei vielen Friedensschlüssen ausschlaggebend.

30 Baumgart (1972), 123. 
fassendem Aufruhr kam; er war mit den Worten des russischen Historikers Gorizontov „an evaluation of the robustness of the imperial structure.“31

Der zeittypische, eher konventionelle Pariser Frieden beendete den ersten ,modernen' Krieg und war nicht von langer Dauer; seine wesentlichen Ergebnisse zumindest bis zum Russisch-Osmanischen Krieg von 1877-78 bzw. dem Berliner Kongress - waren die Neutralisierung des Schwarzen Meeres, die Festlegung, in der Ostsee und im Schwarzen Meer Festungsanlagen, Waffenarsenale etc. nicht wieder aufzubauen, eroberte Gebietsteile zu räumen und Kriegsgefangene auszutauschen..$^{32}$ Die Resultate des Pariser Friedens, so Orlando Figes Urteil, waren insgesamt den hohen Blutzoll „nicht wert“ („,not worthy“), den der Krieg gefordert hatte. Allein Russland hatte mehr als 400.000 Mann verloren und zwar mehr durch Seuchen als durch direkte Kampfhandlungen. ${ }^{33}$

Im Artikel V des Pariser Vertrags wurde festgelegt, dass die Beteiligten „accordent une amnistie pleine et entière à ceux de leurs sujets qui auraient été compromis par une participation quelconque aux évènements de la guerre en faveur de la cause ennemie. “34 Dieser Punkt wurde jedoch, wie mit den Hinweisen auf den krimtatarischen Exodus nach dem Krimkrieg gezeigt, nicht erfüllt. Weder das Osmanische Reich noch das Russländische Imperium gewährten ihren Untertanen eine „vollständige Amnestie“ für vermeintliche oder tatsächliche $\mathrm{Zu}$ sammenarbeit mit dem Gegner, so dass es nicht nur auf der Halbinsel zu Bevölkerungsverschiebungen gewaltigen Ausmaßes im Nachklang des Pariser Friedens gekommen ist: Neben ca. 200.000 muslimischen KrimbewohnerInnen verließen zeitgleich Schätzungen zufolge ca. eine Million Menschen aus dem nördlichen Kaukasus das Zarenreich Richtung Osmanisches Reich, während bulgarische, gagausische und griechische UntertanInnen des Sultans in umgekehrter Richtung aufbrachen. Dies geschah aus wirtschaftlichen und religiösen Gründen, um dem Klima des Misstrauens zu entfliehen, und/oder aus Angst vor Repressionen. Mithin wendeten beide Imperien spezifische, strukturelle Gewaltmechanismen gegen eigene UntertanInnen an, die von Mark Pinson schon vor einigen Jahrzehnten treffend als „demographische Kriegsführung“ - „demographic warfare“ - bezeichnet worden sind. ${ }^{35}$

31 Gorizontov (2012), 66.

32 Vgl. den Text des Pariser Friedens nebst Beilagen in: Traité (1856).

33 Figes (2010), 432.

34 Traité (1856).

35 Pinson (1970). 